\title{
I nfant sleeping and feeding patterning: A cultural perspective on maternal practices
}

\author{
Orly Sarid, Yana Shraga \\ Department of Social Work, Faculty of Humanities \& Social Sciences, Ben-Gurion University of the Negev, Israel \\ Correspondence: Dr. Orly Sarid. Address: Department of Social Work, Faculty of Humanities \& Social Sciences, \\ Ben-Gurion University of The Negev, P.O.B. 653, Beer-Sheva 84105, Israel. Email: orlysa@bgu.ac.il.
}

Received: August 2, 2012

Accepted: September 9, 2012

Online Published: January 7, 2013

DOI : 10.5430/jnep.v3n6p118

URL: http://dx.doi.org/10.5430/jnep.v3n6p118

\section{Abstract}

Aim: To compare practices of Israeli native-born and soviet immigrant mothers in relation to the sleeping and feeding routines of their three, six and twelve month old infants.

Methods: Seventy mothers of infants up to the age of twenty-four months, half native born and half immigrant from the Former Soviet Union (FSU), reported sleep and feeding patterning for their infants at three, six and twelve months. Mothers were matched by socio-demographic characteristics of age, marital status and education. Infant's age and gender were matched as well. Issues addressed included feeding method, adherence to feeding and bedtime schedules, age of infant when mother return to work outside the home.

Results: FSU mothers share a similar feeding and sleeping pattern for their six and twelve month old infants, while native born mothers keep diverse and flexible feeding routines.

Conclusions: We suggest that maternal practices reflect cultural norms. In the case of Soviet culture, a developmentaleducational approach emphasizes the parental role in modifying and adjusting child's behavior, while Israeli culture encourages parental responsiveness to the individual child's specific needs.

\section{Key words}

Mothers, Feeding and bedtime schedules, Cross-cultural

\section{I ntroduction}

This paper will show that Israeli native-born mothers and immigrant mothers from the Former Soviet Union (FSU) adhered to a fixed feeding and sleeping schedule of three month old infants. FSU immigrant mothers share a fixed pattern of scheduling of both feeding and sleep routines for their 6 and 12-month-old infants, whereas Israeli-born mothers follow more diverse practices. Attitudes of native-born Israeli mothers reflect cultural norms of psychological independence and self-sufficiency which are valued as qualities that enhance infant's adaption. FSU immigrant mothers adhere to Soviet -Russian culture norms as conformity, and functional independence as qualities that enhance infant's adaption.

Previous research has shown that differences in maternal perceptions and practices stem from the distinct values and norms of Israeli and Soviet-Russian cultures. Native-born Israeli mothers state that children need autonomy and 
independence in exploring their environment. While mothers from the FSU hold that optimal development is achieved when children are provided with an organized environment in which mothers have a dominant and instructive role ${ }^{[1-3]}$.

Parental child rearing perceptions and practices are shaped in the process of socialization within a cultural context that reflects values about what it means to be a "good" parent ${ }^{[1-3]}$. Parental practices are believed to enhance optimal development and the socialization of the young child into society and to establish culturally valued behaviors ${ }^{[4,5]}$. Thus, certain parental practices can be valued in one culture while a different cultural milieu might view them as maladaptive for the child's well being.

Feeding and sleeping practices are considered to be central ways of caring for infants. Sleep and feeding patterning of infants are one of the earliest forms of culturally determined parental practices. These practices are based on the notion that parental patterning of infant's sleep and feeding enhance the development of the child and reduce sleeping and feeding difficulties ${ }^{[3,5]}$.

Cross-cultural studies have examined infant sleep in respect to sleeping arrangements such as co-sleeping with parents versus letting the infant sleep alone in their own bed and room ${ }^{[3]}$, napping, use of sleep aids, strategies of putting infants to sleep and bedtime rituals ${ }^{[5,6]}$. New and Richman compared the sleeping practices of American and Italian mothers and found that American mothers employ strict strategies for putting their infants to sleep and worry that their infants do not get adequate amounts of sleep. In contrast, Italian mothers do not follow consistent bedtime rituals and are less concerned about the sleep habits of their children. The authors suggest that American mothers understand their maternal role as a regulatory role that aims to enhance order in the infant's life and reduce the risk of developing bad sleeping habits ${ }^{[6]}$.

Like sleep routines, feeding routines also vary. Few comparative studies have examined feeding practices of mothers from different cultural groups. Wolf and colleagues explored maternal attitudes to breastfeeding and bottle intake ${ }^{[3]}$. New and Richman ${ }^{[6]}$ showed that American mothers are flexible in feeding their young infants while Italian mothers keep infants on a strict schedule from a very young age, stating that a feeding routine enables them to satisfy the infant's needs.

The above studies examine parental practices in different countries within distinct cultural contexts. Very few studies have examined the child rearing practices of immigrant mothers in transition from one culture to another. In the process of immigration and acculturation, parents must often contend with divergent child rearing practices and ideologies ${ }^{[7]}$.

In the last decades, over a million immigrants from the FSU (Former Soviet Union) have arrived in Israel ${ }^{[8]}$. A previous study reports that native-born mothers and FSU immigrant mothers have different views of what constitutes good child rearing practice. Unlike Native-born mothers who state that children need autonomy and independence in exploring their environment, and accordingly encourage spontaneous activities of infants. Mothers from FSU hold that optimal development is achieved when children are provided with an organized and educationally simulative environment in which mothers have a dominant and instructive role ${ }^{[9,10]}$. These differences in maternal perceptions and practices stem from the distinct values and norms of Israeli and Soviet-Russian cultures. In Israel, an open, western society, psychological independence, autonomy, assertiveness and self-sufficiency are valued as qualities that enhance adaption. In Soviet -Russian culture, conformity, respect for authority and functional independence are valued qualities ${ }^{[7,11,12]}$.

To the best of our knowledge, no previous cross cultural study in Israel has examined maternal practices with respect to infants sleeping and feeding patterns. Thus, the aim of this study is to compare practices of Israeli native-born and soviet immigrant mothers in relation to the sleeping and feeding routines of their three, six and twelve month old infants.

\section{Methodology}

The study was conducted in the Negev, a region populated by a wide variety of ethnic and socio-economic groups and immigrants ${ }^{[13]}$. It used a cross-sectional design with a sample of 70 participants. Inclusion criteria were having a first child whose age was no more than 24 months; employment before giving birth and return to work after giving birth. 
Thirty-five participants were immigrant mothers from the FSU. Thirty-five native-born Israeli mothers were matched to this sample according to age, education and marital status. Infant age and gender were also matched between the samples. Mothers were recruited using the snowball technique in which one mother suggests another. This technique seems to be appropriate for cross-cultural research and was previously used among people who share the same characteristics; it is usually used when the target population is rare and when the research is cultural sensitive ${ }^{[14-16]}$.

The study was approved by the ethical committee of Ben-Gurion University of the Negev. Informed consent was obtained from all participants. They were told their participation was voluntary and confidentiality was guaranteed. All participants agreed to collaborate and answered a self-report questionnaire.

The questionnaire included:

Feeding methods of the infant -infant feeding methods scale ${ }^{[17]}$ was employed and mothers were asked to indicate (a) exclusive breastfeeding (breast milk only), (b) partial breastfeeding (1-2 bottles of formula per day), (c) bottle-feeding (no breast milk at all). If mothers answered positively to categories (a) and (b) they were asked for the age of their infant when breastfeeding was stopped ${ }^{[18]}$.

Feeding routine at fixed times - Six questions assess feeding schedules during day and night for the 3-month, 6-month and 12-month-old infant. Each question has three possible answers: (a) adhering to a set schedule, (b) not adhering to a set schedule, (c) Other. Cronbach $\alpha$ for these questions was 0.87 .

Putting the infant to sleep at fixed times - Six questions assess sleep schedule during day and night for the 3-month, 6-month and 12-month-old infant. Each question has three possible answers: (a) adhering to a set sleep schedule, (b) not adhering to a set sleep schedule, (c) Other. Cronbach $\alpha$ for these questions was 0.76 .

Return to work - One question asks for the infant's age when mother returned to work.

Socio-demographic data - included infant's age and sex, mother's age, mother's education, country of birth and year of immigration. Subjective assessment of economic status was conducted by one question 19 with three possible answers (a. above average, b. average, c. below average).

\section{Data analysis}

Nominal and ordinal variables are presented by percentages and chi-squared tests. T test analysis was employed to detect possible differences in continuous variables e.g., duration of breastfeeding.

\section{Results}

Mothers from both cultural groups were matched by socio-demographical characteristics: their mean age was 27.5 years old $(\mathrm{SD}=3.54)$. All mothers were married. More than $75 \%$ of the mothers had an academic education. Infants were matched by age and gender: more than half of the infants were boys $(55 \%)$ and their average age was 14.7 months $(\mathrm{SD}=6$ months).

No statistically significant differences in economic status were detected between the two groups of mothers, $88.6 \%$ of mothers from both groups defined their economic status as above average. The average length of time of immigrant mothers in Israel was about 14 years $(\mathrm{SD}=4$ years).

No statistical difference was detected in the main feeding method and the great majority of mothers from both groups $(80 \%)$ reported partial breastfeeding of their infants. The rest used bottle feeding. Breastfeeding duration did not differ 
statistically significantly between the two groups. Mean duration for native-born mothers was 7 months ( $\mathrm{SD}=4$ months) vs. immigrant mothers' mean duration of 8 months ( $\mathrm{SD}=5$ months; $t=0.79$ ( $\mathrm{d} f=55) \mathrm{NS}$ ).

To meet the aim of the study, we compared feeding and sleep practices of native-born and soviet immigrant mothers for their three, six and twelve-month-old infants. Results are presented in Table 1.

Table 1. Mothers' practices regarding set sleep and feeding schedules (Percentages, $\chi^{2}$ test $(\mathrm{d} f=1)$ )

\begin{tabular}{|c|c|c|c|c|}
\hline & & 3 months & 6 months & 12 months \\
\hline \multirow{3}{*}{ Daytime feeding schedule } & Native born & $39 \%$ & $44 \%$ & $53 \%$ \\
\hline & Immigrant mothers & $44 \%$ & $76 \%$ & $82 \%$ \\
\hline & $\chi^{2}$ & NS & $4.8^{*}$ & $4.9^{*}$ \\
\hline \multirow{3}{*}{ Nighttime feeding schedule } & Native born & $26 \%$ & $17 \%$ & $8 \%$ \\
\hline & Immigrant mothers & $38 \%$ & $38 \%$ & $30 \%$ \\
\hline & $\chi^{2}$ & NS & $3.4^{*}$ & $6.7^{*}$ \\
\hline \multirow{3}{*}{ Nighttime sleep schedule } & Native born & $83 \%$ & $80 \%$ & $78 \%$ \\
\hline & Immigrant mothers & $83 \%$ & $93 \%$ & $100 \%$ \\
\hline & $\chi^{2}$ & NS & NS & $6.5^{*}$ \\
\hline
\end{tabular}

${ }^{*} p<05$

No statistically significant differences were found in feeding routines for 3-month-old infants. Thirty-nine percent of native-born mothers and $44 \%$ of immigrant mothers adhered to a feeding schedule during the daytime and $26 \%$ of nativeborn and $38 \%$ of immigrant mothers kept to a schedule in the nightly feeding routine.

With 6-month-old infants, the differences between native-born and immigrant mothers are statistically significant for both day and night feeding routines (daytime schedule: $44 \%$ vs. $76 \%$ and nighttime schedule: $17 \%$ vs. $38 \%$ respectively). Among immigrant mothers, adherence to a feeding schedule during the day and night was high compared with native-born Israeli mothers.

This significant difference persists when infants reach the age of 12 months, with only $53 \%$ of native-born mothers adhering to a daytime schedule as opposed to $82 \%$ of immigrant mothers, and only $8 \%$ keeping to a nighttime schedule in contrast with $30 \%$ respectively.

In sum, immigrant mothers tended to adhere to a fixed feeding schedule in contrast to native-born Israeli mothers.

We found no statistically significant difference in the practices of mothers of 3 and 6-month-old infants for daytime and nighttime sleeping routines. About $83 \%$ of mothers of 3-month-olds from both groups keep to a sleep schedule at night and $75 \%$ keep to a sleep schedule during the day (not shown in table 1). Among mothers of six-month-old $75 \%$ of native-born and immigrant mothers maintain a daytime sleep schedule (not shown in table 1) and $80 \%$ of native-born and $93 \%$ of immigrant mothers maintain a sleep schedule at night, as shown in table 1.

No statistically significant differences were found between the two groups with respect to day time sleep schedule of twelve-month-old infants. However a statistically significant difference was found for mothers of twelve-month-old infants, with all immigrant mothers adhering to a predetermined structured routine for putting their 12-month- old infants to sleep for the night, in contrast to only $78 \%$ of native-born Israeli mothers.

\section{Discussion}

In accordance with the aim of this study, no statistically significant differences were found between the two cultural groups regarding their fixed time feeding and sleeping schedule of three month old infant. The great majority of mothers 
from both groups adhered to a fixed sleep schedule. A possible explanation for the similarities in feeding routine may point to a "universal tendency" of mothers to respond and attend flexibly to their infants needs in the first months after birth ${ }^{[20,21]}$. Our findings are consistent with previous studies that have shown that mothers from traditional and industrialized societies feed their very young infants frequently and do not adhere to a fixed and rigid routine of feeding ${ }^{[22-24]}$.

The great majority of our participating mothers from both cultural groups kept a set daily and nightly sleep routine with their three months old infants. An earlier study found that parents do not adhere to a strict set of behaviors in putting their very young infants to sleep, but tend rather to flexibly adapt to them ${ }^{[25]}$. Our own finding supports Doering Runquist and colleagues ${ }^{[26]}$ work which suggests mothers tend to establish sleeping routines for their very young infants as a way to preserve energy, stabilize mood and prevent fatigue.

Statistically significant differences were found between immigrant and native- born mothers of six and twelve-month-olds regarding daytime and nighttime scheduled feedings with immigrant mothers tending to maintain a stricter schedule than their native-born counterparts. In addition, a significant statistical difference was found between immigrant mothers and native-born mothers in their adherence to a set schedule for putting their 12-month-old infants to sleep for the night. A possible explanation is that these differences in the implementation of set schedules for older infants may reflect cultural norms and parental child care practices which are prevalent in Israeli as opposed to Soviet-Russian culture ${ }^{\text {[9, 27-29]. }}$. Previous studies have shown that the parental style of immigrant parents from FSU is characterized by authority with less consideration of the child's wishes and emotional needs ${ }^{[9,30]}$. FSU immigrant's mothers consider themselves responsible for their children's developmental-educational processes and tend to create structured learning stimuli in an organized environment ${ }^{[9,31]}$. The developmental-educational approach in Soviet-Russian culture emphasizes the parent's role in modifying and adjusting the child's behavior from infancy ${ }^{[31]}$. Although the immigrant mothers participating in the current study have lived in Israel for about 14 years, it is likely that some of the Soviet-Russian norms regarding infant care have endured. As reported in a previous study, child rearing norms and values manifest even among the second generation of immigrants with Soviet-Russian background ${ }^{[32]}$. Our current results corroborate these ideas and suggest that keeping structured and set routines for both feeding and putting infants to bed enables immigrant mothers to optimize care for their infants. In contrast, native-born mothers seek to establish a sense of independence among their infants. This is in keeping with western society's standards which encourage parents to adapt their practices and behaviors to the specific needs of their infant ${ }^{[6,9,28]}$.

All in all, it is interesting to note that FSU immigrant mothers share a similar pattern of scheduling of both feeding and sleep routines for their 6 and 12-month-old infants, whereas Israeli-born mothers follow diverse practices. They keep to a schedule for putting their 6 and 12-month-old infants to sleep but feed them in a more flexible way. Our findings regarding the diverse practices of Israeli-born mothers are in line with a previous study conducted among American mothers. American mothers adhered to strict sleeping practices and flexible feeding routines for their infants ${ }^{[6]}$. This complex pattern was explained as a way of strengthening the infant's independence while at the same time regulating eating habits.

\section{Limitations of study}

The current study has several limitations that need to be addressed. First, all of the data in this study was collected from self reports by the participants and more objective instruments were not employed. Since the questions were retrospective in nature, it is possible that the mother's recollections were not accurate. However this method of research is commonly used by other researchers and yields good results ${ }^{[3,33]}$. Second, the sample size is relatively small and yet, similar sample sizes have been used in the past and have obtained statistically significant differences between cultural groups ${ }^{[6,33]}$. In the future it will be important to conduct similar studies with a larger and more diverse sample. Third, our participants were educated mothers from a relatively high socio-economic status. Future studies that engage mothers from a lower socio-economic status are necessary. Taking in account the above limitations, our ability to generalize the findings to other 
groups of mothers is compromised. However, the findings of this study seem to indicate that cultural norms influenced the adherence of mothers to a distinct set of infant feeding and sleeping practices.

\section{Conclusion}

The results of this study may provide educators and clinicians with knowledge and enhance professional awareness in respect to mother care practice in multicultural societies. Parental practices which are in accordance with the prevailing cultural norms are valued as optimal for child development. Thus, when caring practices do not conform to those maintained by the culture of the majority, stigmatization and stereotyping may cause disagreement between parents and social agents. Attributing differences to the parents' former cultural context promotes a less pathological perspective. This type of understanding may encourage professionals to be more empathic and less judgmental of parents and may consequently help them to employ more effective practices when parents need it ${ }^{[34]}$.

Future comparative studies of parental perceptions from different cultural groups are required to examine other infant care practices in order to provide further knowledge to educators and clinicians and improve appropriate services in multicultural societies.

\section{Acknowledgements}

The research was conducted with a grant from the Rotter Fund, Maccabi Health Care Services.

\section{References}

[1] Fuchs-Shabtai A, Blank S. Too good parents. Or Yehuda: Dvir, (Hebrew), 2004.

[2] Roer-Strier D. Reducing risk for children in changing cultural contexts: recommendations for intervention and training. Child abuse Negl. 2001; 25: 231-48. http://dx.doi.org/10.1016/S0145-2134(00)00242-8

[3] Wolf A, Lozoff B, Latz S, Paludetto R. Parental theories in the management of young children's sleep in Japan, Italy, and the United States. In: Harkness S, Super C. eds, Parents' cultural belief systems, New York: Guilford Press. 1996; $364-84$.

[4] Forehand R, Kotchick BA. Cultural diversity: A wake-up call for parent training. Behav Ther. 1996; 27: 187-206. http://dx.doi.org/10.1016/S0005-7894(96)80014-1

[5] Jenni OG, O'Connor BB. Children's sleep: An interplay between culture and biology. Pediatrics. 2005; 115: 204-15. PMid:15866854 http://dx.doi.org/10.1542/peds.2004-0815B

[6] New RS, Richman AL. Maternal beliefs and infant care practices in Italy and the United States. In: Harkness S, Super CM, eds. Parents' cultural belief systems: Their origins, expressions, and consequences. New York: Guilford Press, 1996; 385-404.

[7] Roer-Strier D. Coping strategies of immigrant parents: Direction for family therapy. Fam Process. 1996; 35: 363-76. http://dx.doi.org/10.1111/j.1545-5300.1996.00363.x

[8] Central Bureau of Statistics. Statistical Abstract of Israel: 59, 2008.

[9] Roer-Strier D, Rosenthal MK. Socialization in changing cultural contexts: a search for images of an adaptive adult. Soc Work. 2001; 46: 215-228. PMid:11495367 http://dx.doi.org/10.1093/sw/46.3.215

[10] Sarid O, Shraga Y. Perceptions and attitudes towards infant's physical health and caring: Immigrants and native born mothers. World Acad Sci Eng Technol. 2009; 55: 216-21.

[11] Jukova A. A particular method for infant development. Moscow: Ulma Press (Russian), 2002.

[12] Martinov S. Be-well, child. A-factoria Press: Ekatirenburg, (Russian), 2006.

[13] Negev Development Authority. Statistical yearbook of the Negev. Beer Sheva: Israel (Hebrew), 2007.

[14] Bloch A. Carrying out a survey of refugees: Some methodological considerations and guidelines. J. Refug Stud. 1999 ; $12: 367-83$. http://dx.doi.org/10.1093/jrs/12.4.367

[15] Bloch A. Methodological challenges for national and multi-sited comparative survey research. J. Refug Stud. $2007 ; 20: 230-47$. http://dx.doi.org/10.1093/jrs/fem002

[16] Liamputtong P. On childrearing and infant care: A cross-cultural perspective. In Liamputtong P ed. Infant feeding practices: A cross-cultural perspective New York: Springer, 2007; 3-29. 
[17] Labbok M, Krasovec K. Toward consistency in breastfeeding definitions. Stud Fam Plann.1990; 21: 226-30. http://dx.doi.org/10.2307/1966617

[18] Donath S, Amir L, The ALSPAC Study Team. Relationship between prenatal infant feeding intention and initiation and duration of breastfeeding: a cohort study. Acta Paediatr. 2003; 92: 352-56. PMid:12725552 http://dx.doi.org/10.1111/j.1651-2227.2003.tb00558.x

[19] Hayo B, Seifert W. Subjective economic well-being in Eastern Europe. J. Econ Psycho. 2003; 24: 329-48. http://dx.doi.org/10.1016/S0167-4870(02)00173-3

[20] Mirsky J. Mothers in Russia and Israel. In Feronni A ed, Motherhood: Psychoanalytic perspective and from another viewpoint Jerusalem: Kibbutz Hameuchad, Van Leer Institute (Hebrew). 2009; 197-214.

[21] Stern DN, Bruschweiler-Stern N, Freeland A. Birth of a mother: How the motherhood experience changes you forever. New York: Perseus Books, 1998.

[22] Hodges EA, Kindermann T, Houck GM. Reliability of the Nursing Child Assessment of Feeding Scale during toddlerhood. Issues Compr Pediatr Nurs. 2007; 30: 109-30. PMid:17885829 http://dx.doi.org/10.1080/01460860701525204

[23] Okechukwu AA, Okolo AA. Exclusive breastfeeding frequency during the first seven days of life in term neonates. Niger. Postgrad. Med. J. 2006; 13: 309-12. PMid:17203121

[24] World Health Organization. Evidence for the ten steps to successful breastfeeding. WHO/CHD/98.9. Division of Child and adolescent health and development. Geneva, 1998. Available from: www.who.int/child_adolescent_health/documents/9241591544/en/

[25] Mindell JA, Sadeh A, Wiegand, B, How TH, Goh D. Cross-cultural differences in infant and toddler sleep, Sleep Med. 2010; 11: 274-80. PMid:20138578 http://dx.doi.org/10.1016/j.sleep.2009.04.012

[26] Doering Runquist JJ, Morin K, Stetzer FC, Severe fatigue and depressive symptoms in lower income urban postpartum women. Western. J. Nurs. Res. 2009; 31: 599-612. PMid:19641094 http://dx.doi.org/10.1177/0193945909333890

[27] Horowitz T. The Soviet man in an open society. In: Horowitz T, ed. The Soviet man in an open society, Lanham, MD: University Press of America, 1989; 5-18.

[28] Slonim-Nevo V, Shraga Y. Family intervention among Soviet Immigrants. In: Rabin C, ed. Being different in Israel: ethnicity, gender and therapy, Ramot: Tel Aviv. 1999; 121-51.

[29] Shor R. Significance of cultural factors in assessing risk factors of children in families from the Former Soviet Union. In Leshem, E, Roer-Strier D eds. Cultural diversity as a challenge to human services, Jerusalem: Magnes Press. (Hebrew), 2003.

[30] Slonim-Nevo V, Shraga Y, Mirsky J. A culturally sensitive approach to therapy with immigrant families: The case of Jewish emigrants from the Former Soviet Union. Fam. Process. 1999; 38: 445-63. PMid:10668622 http://dx.doi.org/10.1111/j.1545-5300.1999.00445.x

[31] Kozulin A, Gindis B, Ageyev V, Miller SM. Vygotsky's educational theory in cultural context. Cambridge, United Kingdom: Cambridge University Press, 2003. http://dx.doi.org/10.1017/CBO9780511840975

[32] Slonim- Nevo V, Sharaga Y. Psychological and social adjustment of Russian-born and Israeli-born Jewish adolescents. Child. Adolescent. Soc. Work. J. 2000; 17: 455-77. http://dx.doi.org/10.1023/A:1026483828067

[33] Leach P, Barnes J, Nichols M, Goldin J, Stein A, Sylva K, Malmberg LE. Child care before 6 months of age: a qualitative study of mothers' decisions and feelings about employment and non-maternal care. Infant. Child. Dev. 2006; 15: 471-502. http://dx.doi.org/10.1002/icd.473

[34] Campinha-Bacote J. The process of cultural competence in the delivery of healthcare services: a model of care. J. Transcult. Nurs. 2002; 13: 181-84. PMid:12113146 http://dx.doi.org/10.1177/10459602013003003 